
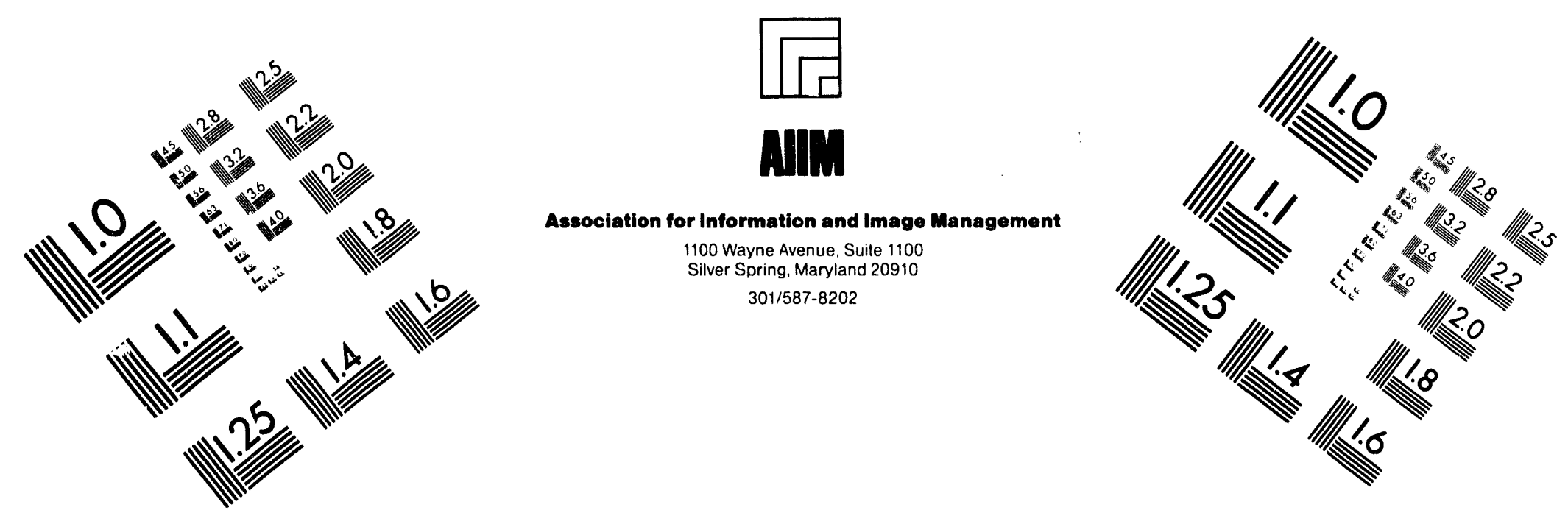

Centimeter

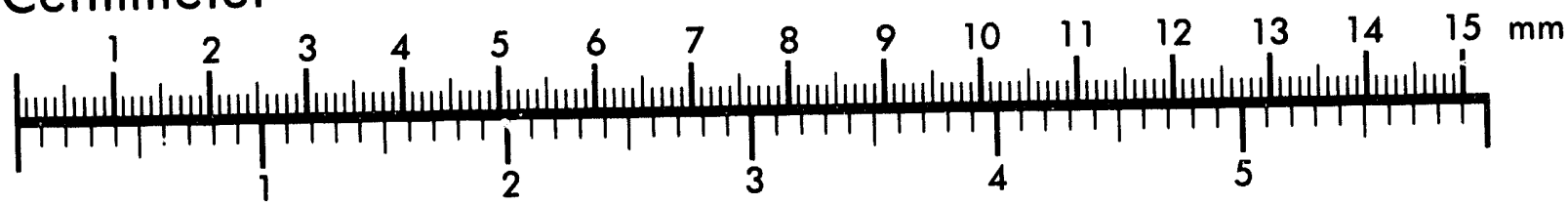
Inches
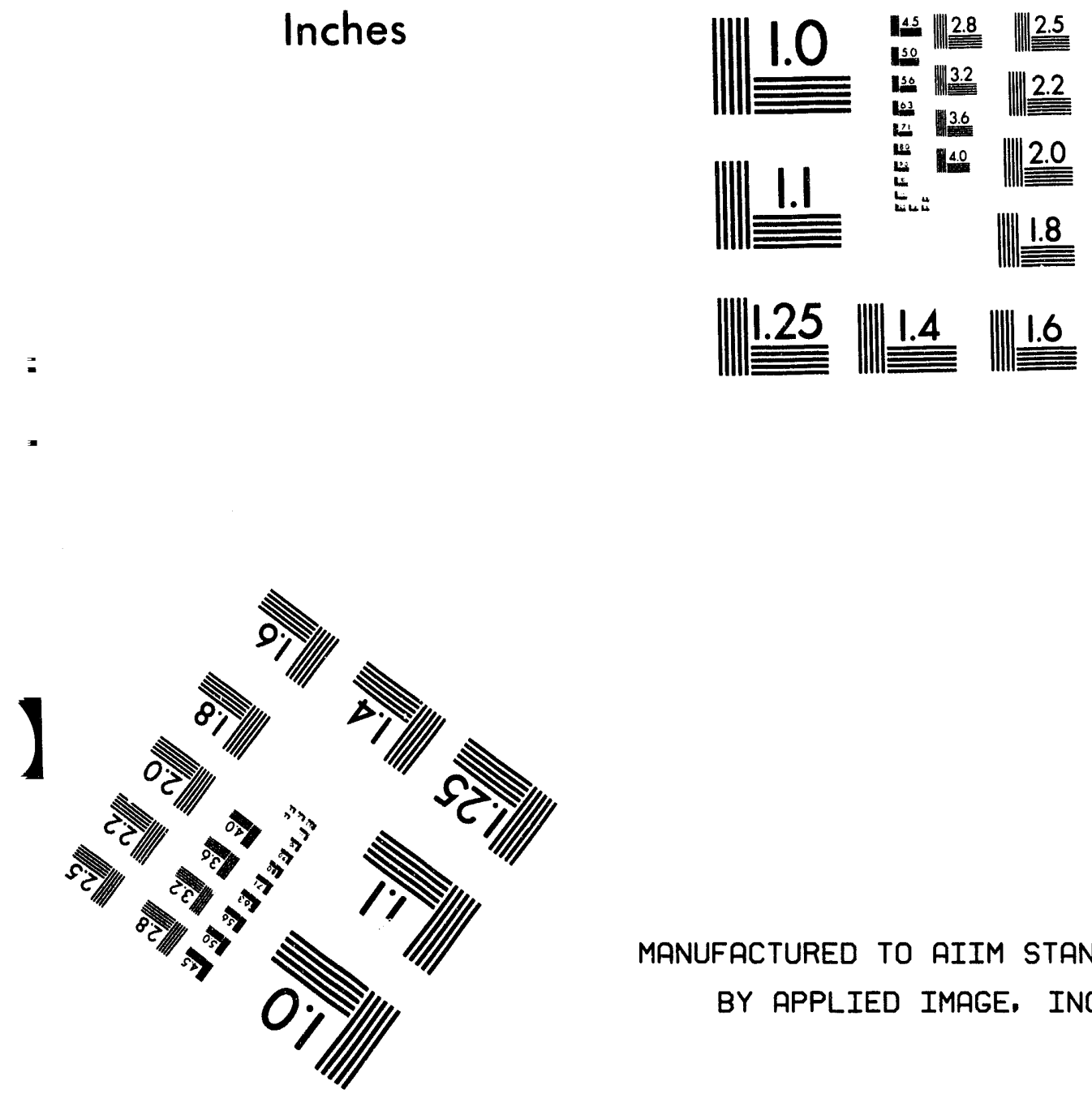

MANUFACTURED TO AIIM STANDARDS

BY APPLIED IMAGE, INC.

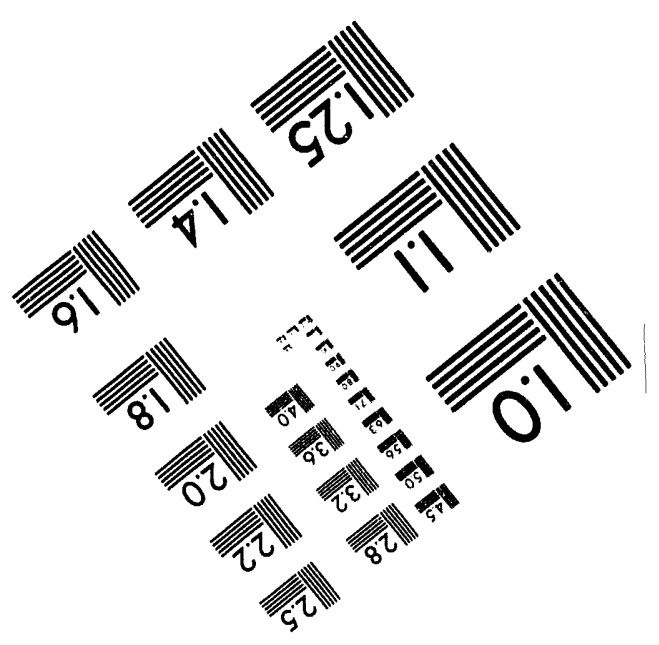



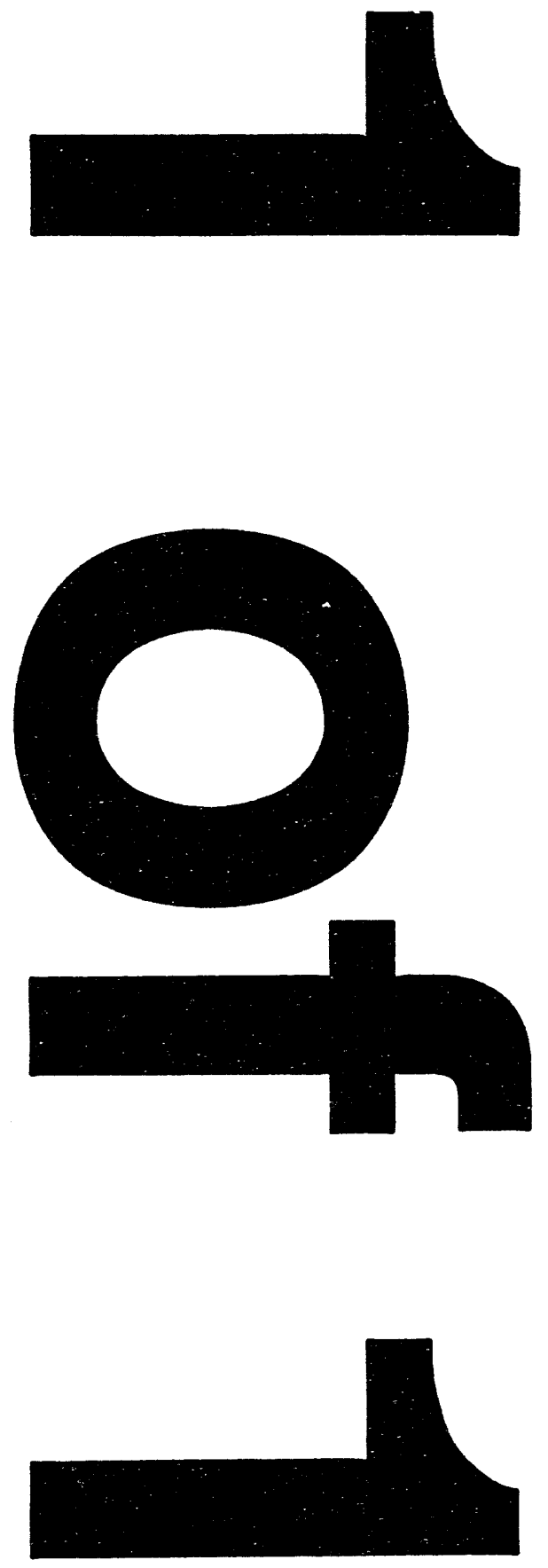


\title{
CONF-931108--108
}

Note: This is a preprint of a paper submitted for publication. Contents of this paper should not be quoted or referred to without permission of the author(s).

For publication in Mechanisms of Thin Film Evolution,

ed. by S. M. Yalisove, C. V. Thompson, and D. J. Eaglesham,

Materials Research Society, Pittsburgh, Pennsylvania,

Fall Meeting of the Materials Research Society, Boston, Massachusetts, November 29-December 3, 1993

\section{SURFACE STRESS, MORPHOLOGICAL DEVELOPMENT, AND DISLOCATION NUCLEATION DURING STRAINED-LAYER EPITAXY}

\author{
D. E. Jesson and S. J. Pennycook \\ Solid State Division \\ Oak Ridge National Laboratory \\ Oak Ridge, Tennessee 37831-6030
}

\author{
J.-M. Baribeau and D. C. Houghton \\ Institute for Microstructural Sciences \\ National Research Council of Canada \\ Ottawa K1A OR6, Canada
}

\section{DISCLAIMER}

\begin{abstract}
This report was prepared as an account of work sponsored by an agency of the United States Government. Neither the United States Government nor any agency thereof, nor any of their employees, makes any warranty, express or implied, or assumes any legal liability or responsibility for the accuracy, completeness, or usefulness of any information, apparatus, product, or process disclosed, or represents that its use would not infringe privately owned rights. Reference herein to any specific commercial product, process, or service by trade name, trademark, manufacturer, or otherwise does not necessarily constitute or imply its endorsement, recommendation, or favoring by the United States Government or any agency thereof. The views and opinions of authors expressed herein do not necessarily state or reflect those of the United States Government or any agency thereof.
\end{abstract}

\author{
SOLD STATE DIVISION \\ OAK RIDGE NATIONAL LABORATORY \\ Managed by \\ MARTIN MARIETTA ENERGY SYSTEMS, INC. \\ under \\ Contract No. DE-ACO5-840R21400 \\ with the \\ U.S. DEPARTMENT OF ENERGY \\ Oak Ridge, Tennessee
}

December 1993

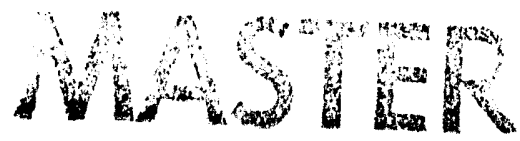




\title{
SURFACE STRESS, MORPHOLOGICAL DEVELOPMENT, AND DISLOCATION NUCLEATION DURING STRAINED-LAYER EPITAXY
}

\author{
D. E. JESSON,* S. J. PENNYCOOK,* J.-M. BARIBEAU,† AND D. C. HOUGHTON` \\ *Solid State Division, Oak Ridge National Laboratory, Oak Ridge, Tennessee 37831-6030 \\ tInstitute for Microstructural Sciences, National Research Council of Canada, Ottawa K1A OR6, \\ Canada
}

\begin{abstract}
Utilizing marker layer experiments and Z-contrast imaging, we have observed the formation of surface cusps during $\mathrm{Si}_{2} \mathrm{Ge}_{1 . x}$ alloy growth. The formation of cusps can be understood in terms of stress-driven surface diffusion, and we consider the large stress build-up at the cusp tip as a potential source for the nucleation of misfit dislocations.
\end{abstract}

\section{INTRODUCTION}

The instability of strained epitaxial thin films to the formation of surface waves or undulations has been appreciated for several years [1-3]. Atoms situated close to the peaks of the undulations can appreciably relax, significantly lowering the stored elastic energy of the film. Provided that the undulation wavelength is sufficiently large, this more than compensates for the associated increase in surface energy.

The peak relaxation is inevitably accompanied by a concentration of stress at the undulation valleys which can be large, even for rather small surface perturbations. Indeed, the valley stress doubles for a sinusoidal surface wave amplitude equal to only one tenth of its wavelength [4]. In this paper, we consider the role of such large surface stresses in the subsequent morphological development of thin films and the potential implications for strain relaxation via the nucleation of misfit dislocations.

\section{MARKER LA YER EXPERIMENTS}

In order to study morphological development in the presence of high surface stresses, we have performed a Si 0.5 Geo.5 MBE growth experiment in which two-monolayer-thick Ge marker layers were deposited at selected intervals [5]. The marker layers were then imaged in cross section using Z-contrast scanning transmission electron microscopy [6] to provide a direct map of the evolving nonequilibrium surface morphology.

A typical experimental result for $\mathrm{Si}_{0.5} \mathrm{Ge} 0.5$ grown at $400^{\circ} \mathrm{C}$ and $2 \mathrm{As}^{-1}$ is contained in Fig. 1. The marker layers appear as bright horizontal lines. Initially, the surface morphology is flat until the film is about $25 \mathrm{~nm}$ thick, where a ripple morphology can be clearly distinguished. This can be understood as a kinetic critical thickness $h_{\mathbf{k}}$ at which the strain-driven morphological development has become significant in comparison with the growth rate. Surface rippling is therefore kinetically inhibited, which is consistent with the far-from-equilibrium growth conditions (for a quantitative model of $h_{k}$, see Ref. 7).

A surprising feature of Fig. 1 is the rapid development of sharp cusp-like features from the surface undulations. These features would appear to be highly metastable, persisting for $20 \mathrm{~nm}$ or so before rapidly smoothing out. The film then grew with a flat surface morphology (within the sensitivity of the experiment) for the remainder of the deposition.

It is interesting to note that the marker layer technique would seem to offer significant advantages for the study of far-from-equilibrium growth shapes in the presence of large surface stresses. In particular, it is possible to maintain a high supersaturation throughout the growth experiment, which is important for high misfit films where large stress concentrations can considerably enhance surface diffusion. Thus, marker layer experiments should faithfully map the far-from-equilibrium growth morphology and avoid uncertainties inherent in conventional "quench and look" approaches. 


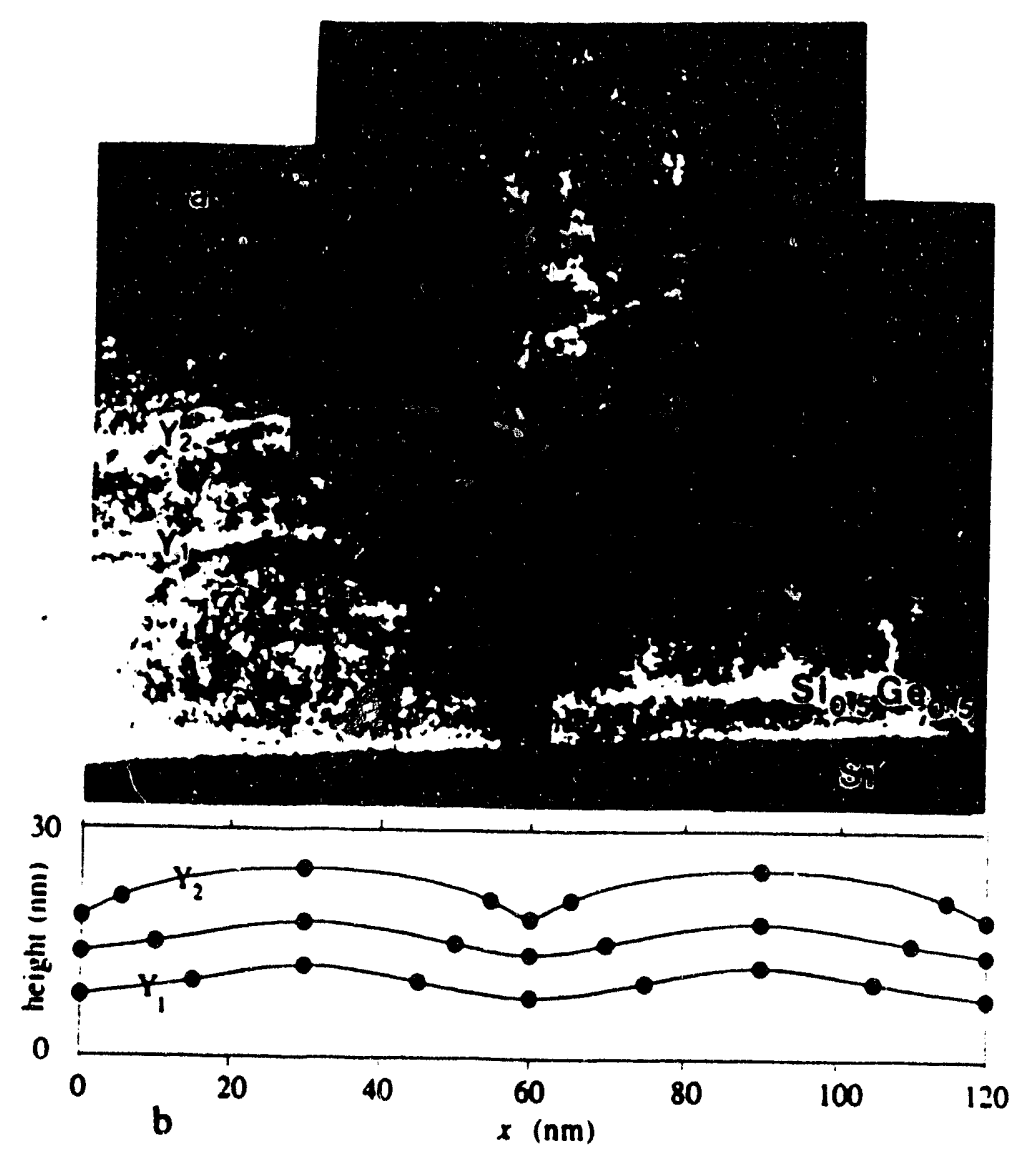

Fig. 1. (a) [110] Z-contrast image of a Sio.sGeo.s alloy grown by MBE at $400^{\circ} \mathrm{C}$ and $2 \AA s^{-1}$. The bright horizontal lines represent 2-monolayer-thick Ge marker layers. The surface profile simulations in (b) correspond to the period of cusp formation in (a) between vertical ordinates $Y_{1}$ and $Y_{2}$ (see Ref. [5]).

\section{ORIGIN OF SURFACE CUSPS}

Given that surface diffusion is driven by gradients in surface chemical potential, the development of a cusp at the valley of a surface wave is rather intuitive $[6,8]$. Consider, for example, a diffusing adatom bonding at the valley of a sinusoidal perturbation in the surface. At this location, the atom experiences the greatest stress concentration and would prefer to migrate to the more relaxed peaks. However, diffusion from the valley to the peaks increases the stress concentration at the valley, which in turn accentuates the rate of migration. It is, therefore, not difficult to anticipate the formation of a cusp. These ideas can be shown to be reasonable using a simple model for cusp development [5]. For example, the surface profile simulations in Fig. 1 qualitatively reproduce all of the experimental features of cusp formation between $Y_{1}$ and $Y_{2}$. Recently, more sophisticated models of surface evolution have also predicted the formation of cusps [9-11]. It is conceivable that these models combined with marker layer experiments could form the basis for the quantitative study of nonlinear surface instabilities.

An interesting prediction of all the surface evolution models (including the simulation in Fig 1) is that upon attaining a critical cusp geometry, the stress concentration at the cusp tip increases rapidly. At this point, the cusp accelerates rapidly into the film via the process of stress-enhanced surface diffusion leading to an interesting comparison between the critical geometry for cusp propagation and the well-known Griffith criterion for the unstable propagation of a crack in a brittle material $[8,9,12]$. This is probably best appreciated when the cusp is under tensile stress, where it has been suggested that the stress-driven morphological instability may in fact precede and initiate the formation of a critical Griffith crack [9]. In compression, a large 
stress concentration must also develop, but this cannot directly lead to fracture of the film. Rather, the likely mechanism of strain relief is the injection of misfit dislocations $[5,8]$, which we consider further in the following section.

\section{NUCLEATION OF MISFTT DISLOCATIONS}

To model dislocation nucleation at a cusp tip, we have previously approximated the cusp stress field by the stress field of a crack [5]. In that analysis, only the dominant tensile component of the crack was considered. Here, we extend the analysis to include the other tensile and shear components and consider the nucleation of partial as well as $60^{\circ}$ half loops. The geometry of half loop nucleation at a cusp tip is represented schematically in Fig. 2. Following Ref. [5], we write the total energy as a function of expanding loop radius $R_{L}$ as,

$$
U\left(R_{L}\right)=R_{L} \frac{b^{2} \mu}{8} \frac{2-v}{1-v} \ln \left[\frac{\alpha R_{L}}{b}\right]-\frac{R_{L} \mu b^{2}}{4} \sin \beta+\frac{\pi R_{L}^{2}}{2} \gamma-C\left(R_{L}\right) \cos \frac{\phi}{2} \sin \phi \sin \beta .
$$

The first term specifies the energy cost of a dislocation of core parameter $\alpha$ and Burgers vector $b$ in a medium of shear modulus $\mu$ and Poisson's ratio $v$. The second term describes the energy gained by the removal of a surface step, $\beta$ being the angle between $b$ and the dislocation line. The energy cost per unit area $\gamma$ of the stacking fault associated with partial dislocations is included in the third term. The fourth term describes the elastic energy released by the loop, where the angle $\phi$ is defined in Fig. 2, and $C\left(R_{L}\right)$ depends on the model used for the radial $(R)$ dependence of the stress field away from the tip. For the Westergaard sharp crack solution [13], we obtain

$$
C\left(R_{L}\right)=2.47 d^{1 / 2} R_{L}^{3 / 2} \mu \frac{1+v}{1-v} \varepsilon b
$$

where $\varepsilon$ is the applied uniaxial stress and $d$ is the crack depth (Fig. 2). From Eq. (1), it is possible to determine the critical cusp depth at which the activation barrier is equal to the available energy for nucleation ( $37 \mathrm{kT}[14])$. This leads to the simple condition for $\mathrm{d}^{\mathrm{c}}$

$$
\sqrt{d^{c} \varepsilon}=A^{\beta}(T, \gamma) \text {, }
$$

where the constant $A^{\beta}$ is dependent on the nature of the dislocation (i.e. $\beta=30^{\circ}, 60^{\circ}$, or $90^{\circ}$ ), stacking fault energy, and temperature. For a given dislocation type, $A \beta$ (T, $\gamma$ can be estimaced from a single energy calculation using Eq. (1). The critical cusp geometry $\mathrm{d}^{c}$ is, therefore, simply related to misfit via Eq. (3), and the results are summarized in Fig. 3 using $A^{30}=0.402$, $A^{60}=0.276$, and $A^{90}=0.177$. The calculation pessimistically assumes a core parameter of 4 for all dislocation types.

An interesting feature of Fig. 3 is that it is energetically more favorable to nucleate a complete $60^{\circ}$ half loop from a cusp rather than a $30^{\circ}$ partial. This is important because the glide plane geometry dictates that it is necessary to nucleate the $30^{\circ}$ partial before the energetically favorable $90^{\circ}$ partial. The nucleation of complete $60^{\circ}$ dislocations is, therefore, favored in this case for atomically sharp cusps. It is interesting to compare half loop nucleation at cusps with half loop nucleation at flat surfaces, where assuming a step is removed, it is always favorable to inject a complete $60^{\circ}$ dislocation before a $30^{\circ}$ partial. However, unlike the cusp, the energy barrier for the nucleation of a $90^{\circ}$ partial is only lower than the $60^{\circ}$ half loop barrier down to $1 \%$ misfit. At this point, the critical radius is very large, and the stacking fault energy correspondingly high. Note that in the case of a critical cusp geometry, the critical radius is always small, rendering the stacking fault energy less important.

The $60^{\circ}$ curve in Fig. 3 suggests that dislocation nucleation at the tip of an atomically sharp cusp is only likely for misfits somewhat larger than $1 \%$. This is because the scale of morphological development in this system at $1 \%$ misfit is only of the order of a few hundred Ángstroms [15]. 


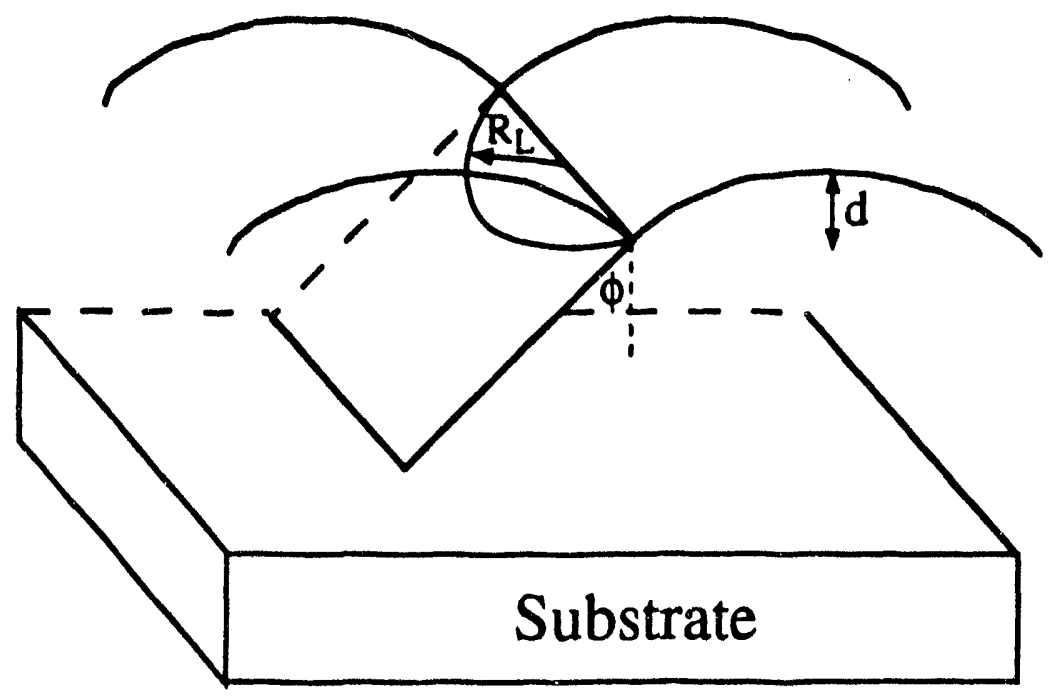

Fig. 2. The geometry of dislocation half loop emission at the tip of a surface cusp (see text).

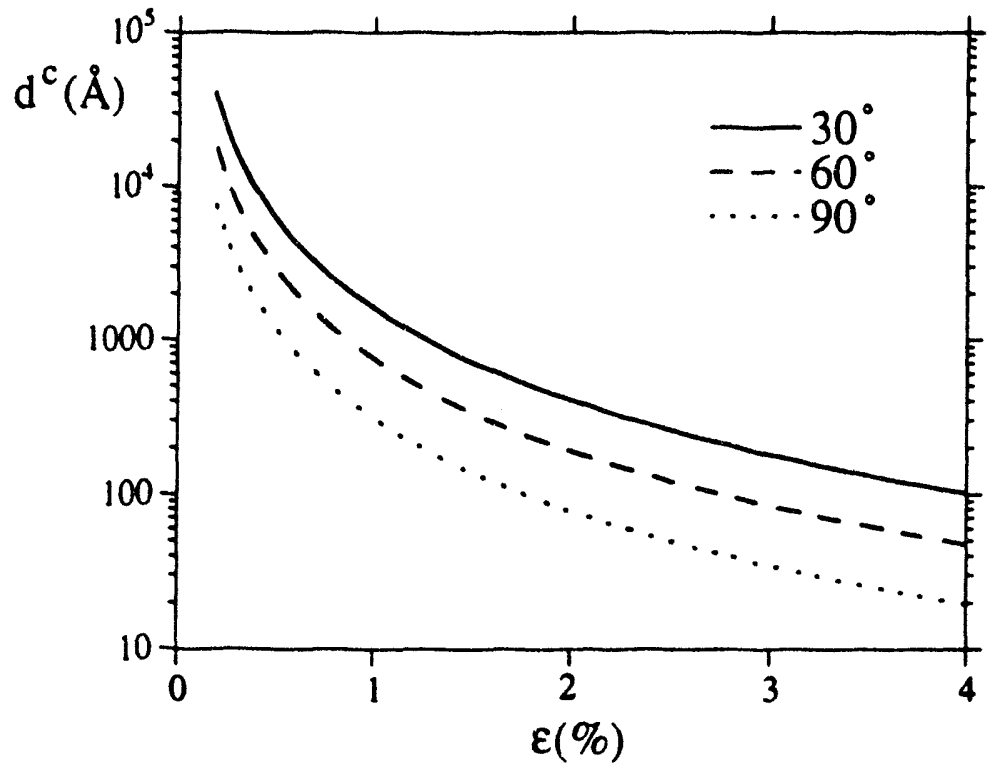

Fig. 3. Critical cusp depth $d^{c}$ required to nucleate $30^{\circ}, 60^{\circ}$, and $90^{\circ}$ dislocations as a function of misfit $\varepsilon$ assuming an atomically sharp cusp. The curves are evaluated using Eq. (3).

The utilization of a sharp crack model for dislocation emission at a cusp tip can provide useful insight into the dislocation emission process. However, such a model is only valid if the critical radius $R_{\mathcal{c}}$ is significantly greater than the cusp radius of curvature $p$ and appreciably less than the cusp depth $d c$. Clearly, this is not true for very large misfits close to $4 \%$ involving small $\mathrm{d}$ and $\mathrm{R}_{\mathrm{c}}$. Furthermore, it is clear from our images that at $2 \%$ misfit the cusps are not atomically sharp so that the sharp crack solution is not strictly applicable. When the critical loop radius is of the order of the tip radius of curvature $\rho$, a more suitable model is the blunt crack approximation [12] giving

$$
C\left(R_{L}\right)=2 \frac{1+v}{1-v} \varepsilon \mu b d^{1 / 2} \int_{0}^{R_{L}}\left[\rho+4\left(R_{L}^{2}-x^{2}\right)^{1 / 2}\right]^{1 / 2}-\rho^{1 / 2} d x .
$$


The additional term

$$
E\left(R_{L}\right)=-\pi R_{L}^{2} \mu \frac{1+v}{1-v} \varepsilon b
$$

associated with the mean elastic energy released by the loop must also be added to the right side of Eq. (1) in the blunt crack model.

The blunt crack model conveys the important point that the critical cusp depths estimated from the sharp crack model in Fig. 3 are likely to be significant overestimates. Although the absolute stress concentration is naturally reduced at a blunt tip, the resolved shear stress field is significant over a larger region of the glide plane than in the case of a sharp crack. This can appreciably assist the nucleation process. To illustrate this, let us consider the experimental geometry in Fig. 1 in more detail. The cusp depth is approximately $75 \AA$, although it is more difficult to estimate the sharpness of the tip as the marker layers appear interdiffused in this highly stressed region. Figure 3 suggests that we require a "sharp cusp" of depth $190 \AA$ for the nucleation of a $60^{\circ}$ half loop. This reduces to $145 \AA$ for a reasonable reduction in the core parameter to $\alpha=3$. The blunt crack model, however, indicates that nucleation will occur at $d=75 \AA$ provided $\rho=15 \AA$ and $\alpha=3$. The latter result would appear to be in particularly good agreement with the experimental geometry in Fig. 1.

A blunted cusp tip migh: also be capable of operating as a dislocation nucleation source down to low misfits. For example, the undulating morphology of amplitude $400 \AA$ observed at a $1 \%$ misfit in Ref. [15] would require $p=10 \AA(\alpha=3)$. Although such cusps might eventually develop during growth, we would anticipate increasing competition from heterogeneous nucleation sources at lower misfits.

The nucleation of misfit dislocations at the tip of a cusp is clearly connected with the question of ductile vs brittle behavior and the stability of cleavage cracks. We have recently become aware of the analysis by Rice and Thomson [16] who studied the problem of the emission of a dislocation loop from a sharp crack in a manner very similar to Ref. [5], and they obtained a result essentially identical to Eq. (1). It is interesting that the concept of crack blunting by dislocation emission, which is of relevance to crack stability, might also be relevant to the case of atomically sharp cusps if the emitted loop has a Burgers vector component normal to the cusp plane. This effect would tend to turn off the cusp source and would require further sharpening by stress enhanced surface diffusion before the emission of additional loops. Furthermore, several loops emitted on a given (111) plane will tend to produce a dislocation pileup at the substrate, creating a back stress at the cusp tip source. If the effective source stress is smaller than the threshold stress required to generate a single loop, then the source will shut down. It is conceivable that the cusp will then continue to develop (and possible sharpen) during deposition, emitting further loops on different (111) planes. This role as a multiple source might explain why the cusp geometry appears to persist for $20 \mathrm{~nm}$ or so before rapidly flattening out as the mean strain in the film is eventually relieved.

\section{CONCLUSIONS}

We have imaged surface cusps during the growth of a strained Sio.sGe0.5 film. The origin of the cusps can be explained in terms of stress-driven surface diffusion, and we have demonstrated that cusp tips provide likely sites for dislocation nucleaticn over a wide range of misfit.

\section{ACKNOWLEDGMENTS.}

We would like to thank S. L. Carney, T. C. Estes, asi J. T. Luck for technical assistance. This research was sponsored by the Division of Materials Sciences, U.S. Department of Energy, under contract DE-AC05-84OR21400 with Martin Marietta Energy Systems, Inc. 


\section{REFERENCES}

[1] R. J. Asaro and W. A. Tiller, Metall. Trans. 3, 1789 (1972).

[2] M. A. Grinfeld, Sov. Phys. Dokl. 31, 831 (1986).

[3] D. J. Srolovitz, Acta. Metall. 37, 621 (1989).

[4] H. Gao, J. Mech. Phys. Solids 39, 443 (1991).

[5] D. E. Jesson, S. J. Pennycook, J.-M. Baribeau, and D. C. Houghton, Phys. Rev. Lett. 71, 1744 (1993).

[6] S. J. Pennycook and D. E. Jesson, Phys. Rev. Lett. 64, 1943 (1990).

[7] B. J. Spencer, P. W. Voorhees, and S. H. Davies, J. Appl. Phys. 73, 4955 (1993).

[8] D. E. Jesson, S. J. Pennycook, J.-M. Baribeau, and D. C. Houghton, MRS Symp. Proc. 312 , 47 (1993).

[9] W. H. Yang and D. J. Srolovitz, Phys. Rev. Lett. 71, 1593 (1993).

[10] B. J. Spencer and D. I. Meiron, submitted to Acta. Metall.

[11] H. Gao (private communication).

[12] See, for example, A. S. Tetelman and A. J. McEvily, Fracture of Structural Materials, John Wiley and Sons, Inc., New York, London, Sydney, 1967.

[13] See, for example, J. F. Knott, Fundamentals of Fracture Mechanics, John Wiley and Sons, New York, Toronto, 1973, p. 57.

[14] S. V. Kamat and J. P. Hirth, J. Appl. Phys. 67, 6844 (1990).

[15] A. J. Pidduck, D. J. Robbins, A. G. Cullis, W. Y. Leong, and A. M. Pitt, Thin Solid Films 222, 78 (1992).

[16] J. R. Rice and R. Thomson, Philos. Mag. 29, 73 (1974). 

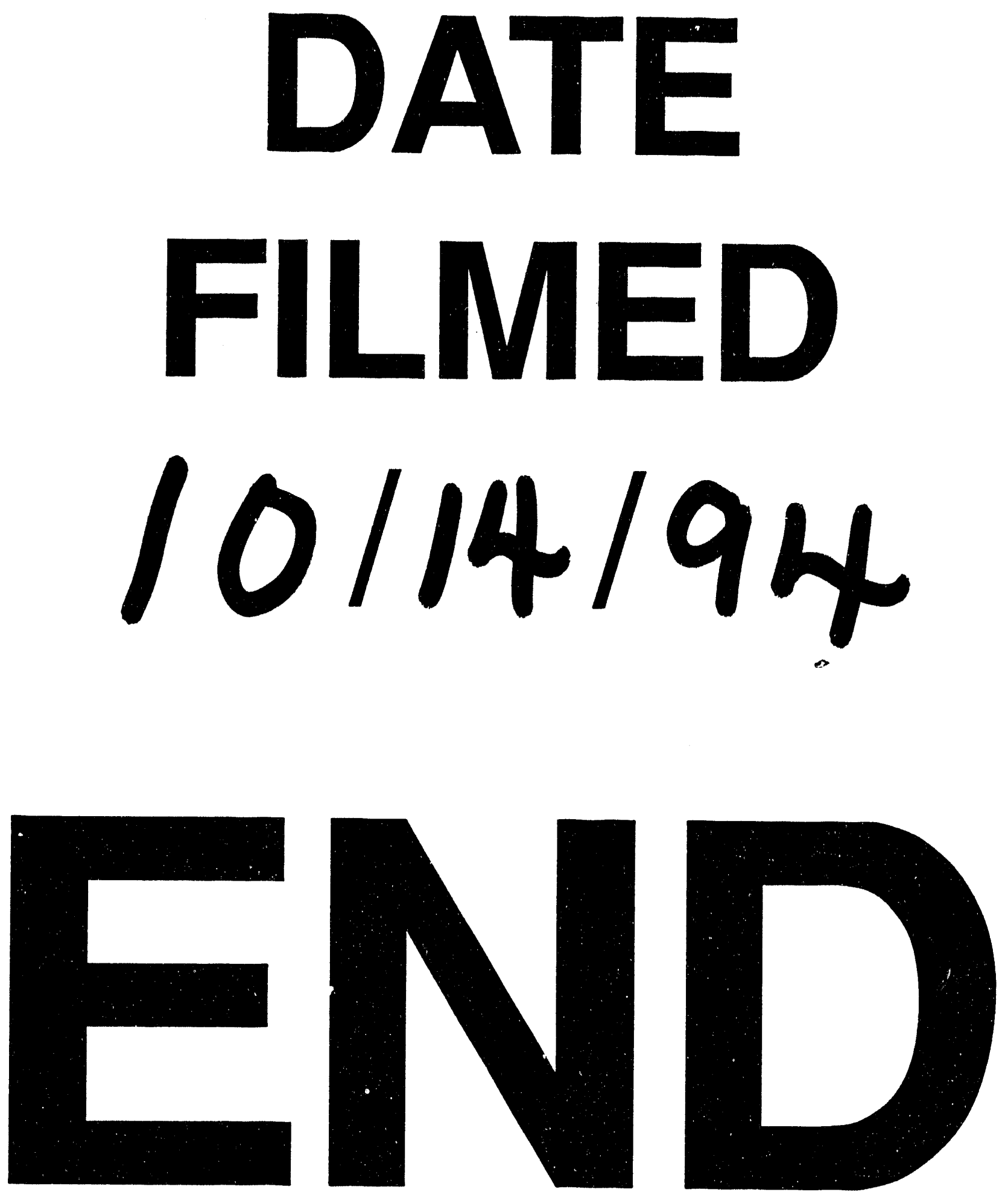
